\title{
Effects of Different Concentrations of PM2.5 on the Expression of the Nasal Mucosa, Olfactory Bulb, and Related Inflammatory Factors in Rats
}

Zhiming Jiang ( $\sim 1462213483 @ q q . c o m)$

Anhui University of Traditional Chinese Medicine - Meishan Road Campus: Anhui University of Traditional Chinese Medicine https://orcid.org/0000-0003-

3117-6263

Lina Zhao

Anhui University of Traditional Chinese Medicine - East Campus: Anhui University of Traditional Chinese Medicine

Xiaojia Li

Anhui University of Traditional Chinese Medicine - East Campus: Anhui University of Traditional Chinese Medicine

Zijian Wu

Anhui University of Traditional Chinese Medicine - Meishan Road Campus: Anhui University of Traditional Chinese Medicine

Ling Hu

Anhui University of Traditional Chinese Medicine - East Campus: Anhui University of Traditional Chinese Medicine

\section{Research}

Keywords: moxa smoke, nasal mucosa, olfactory bulb, inflammation, PM2.5

Posted Date: June 11th, 2021

DOI: https://doi.org/10.21203/rs.3.rs-582804/v1

License: @ (i) This work is licensed under a Creative Commons Attribution 4.0 International License. Read Full License 


\section{Abstract}

Background

Although the damaging effect of inhaled smoke on the respiratory system has been widely reported, there are relatively few studies on the effects of inhaled smoke on the nasal mucosa and olfactory bulb. In this study, we observed the activities of adult rats chronically exposed to different concentrations of PM2.5 and analyzed its effects on the olfactory function and the expression of inflammatory factors in the serum, nasal mucosa, and olfactory bulb of rats.

Methods

SD rats were randomly divided into normal control, low-smoke, middle-smoke, and high-smoke groups. The groups exposed to different concentrations of PM2.5 smoke were treated with smoke environment intervention for $4 \mathrm{~h}$ twice daily for 90 days, while the normal control group was fed routinely,After $90 \mathrm{~d}$, the behavior of in the food-seeking glomeruli of rats was analyzed. The contents of IL-1 $\beta$, IL- 6 , and TNF- $\$ in the serum, nasal mucosa, and olfactory bulb of rats were determined by ELISA, and the correlation between them was analyzed. taken for HE staining and TUNEL detection to observe the pathological damage of the nasal mucosa and olfactory bulb.

Results

After a series of tests, we found that Long-term exposure to the low, medium, and high concentrations of moxa smoke can cause pathological changes in the nasal mucosa, increase the number of apoptotic cells in the nasal mucosa and olfactory bulb, and increase the expression of IL- $1 \beta$, IL-6, and TNF- $\$ in the serum, nasal mucosa, and olfactory bulb. Simultaneously, The behavior of the rats also changed. The rats in the medium and high concentrations of moxa smoke searched for food longer, traveled shorter distances and slowed down.

Conclusion

Long-term exposure to medium and high concentrations of moxa smoke can decrease the olfactory sensitivity of rats.

\section{Background}

Odor is the main sensory function that plays an important role in recognition, alarm, appetite, and emotion. This function is mainly performed by the olfactory system. Chemical molecules can be transmitted to the olfactory nerve through the transformation of olfactory cells in the olfactory mucosa. The olfactory nerve will continue to transmit the signal to the brain in the olfactory bulb. The nasal mucosa and olfactory bulb not only play an important role in regulating the sense of smell in the olfactory system ${ }^{[1]}$ but also in physiological functions such as appetite regulation, lactation, response to adverse environments, and social interaction ${ }^{[2-3]}$. The nasal mucosa and olfactory bulb contain several afferent sensory cells and can be directly exposed to adverse external stimuli, such as smoke. Therefore, the damage of nasal mucosa and olfactory bulb caused by long-term inhalation of smoke may affect its sensory function. Injuries due to smoke inhalation are mainly caused by the inhalation of byproducts of combustion, such as PM2.5, carbon monoxide, cyanide, and other toxic gases

${ }^{[4-5]}$. The damage to the respiratory system due to smoke inhalation has been widely reported ${ }^{[6]}$, but there are relatively few studies on the olfactory and central nervous system caused by long-term smoke inhalation. Long-term smoke inhalation has a greater impact on the nasal mucosa and olfactory bulb ${ }^{[7-9]}$. Smoke will first enter the body directly through the nasal mucosa and olfactory bulb to affect the olfactory system. A research group observed the content of fine particles (PM2.5) at a moxibustion clinic in the early stage ${ }^{[10]}$, based on which the different concentrations of moxa smoke for animal experiments were determined, and the effects of different concentrations of PM2.5 moxa smoke on the heart, lung, and brain function of rats were observed ${ }^{\text {[11-12] }}{ }^{\text {Based on the }}$ previous experiment, the effects of moxa smoke with different concentrations of PM2.5 on rats were observed through a 90-day smoke environment intervention experiment. The behavior of buried food pellet test (BFPT) and the pathological changes of the nasal mucosa and olfactory bulb were evaluated, and the changes in and correlation between IL-1 $\beta$, IL- 6 , and TNF-a in serum, nasal mucosa, and olfactory bulb were analyzed. The effects of different concentrations of PM2.5 on the olfactory function of rats are discussed.

\section{Materials And Methods}

1.1 Animals and groups.

A total of 40 healthy normal SD rats, 20 male and 20 female, were selected for the study. The mean body weight was (190 \pm 10$) \mathrm{g}$. The rats were provided by the Anhui Experimental Animal Center, animal license number: SCXK (Wan) 20190003. The animals were fed adaptively in the animal room of the key laboratory at the Anhui Provincial Department of Education for three days. The temperature was $(27 \pm 1)^{\circ} \mathrm{C}$ and the humidity was $(55 \pm 5) \%$. The bedding, feed, and drinking water were replaced every day. The experimental process strictly followed the "guiding opinions on being kind to Experimental Animals" issued by the Ministry of Science and Technology of the People's Republic of China (2006). The rats were randomly divided into normal control group, low PM2.5 concentration moxa smoke environment group (low-smoke group), medium PM2.5 concentration moxa smoke environment group (middle-smoke group), and high PM2.5 concentration moxa smoke environment group (high-smoke group), with 10 rats in each group. According to a previous study, the PM2.5 concentration of moxa tobacco in the low-smoke, middle-smoke, and high-smoke groups was controlled at $0.11 \pm 0.05 \mathrm{mg} / \mathrm{m}^{3}, 0.23 \pm 0.05 \mathrm{mg} / \mathrm{m}^{3}$, and $0.53 \pm$ $0.05 \mathrm{mg} / \mathrm{m}^{3}$, respectively.ELISA kits for IL-1 $\beta$, IL-6, and TNF-a (Wuhan Genmei Technology Co., Ltd., batch number: GR2020-05), three years 3:1 Chen moxa (18 $\mathrm{mm} \times 200 \mathrm{~mm}$ Shanghai Taicheng Science and Technology Development Co., Ltd.), three cuboid plexiglass moxibustion boxes with a volume of $100 \mathrm{~cm} \times$ $80 \mathrm{~cm} \times 125 \mathrm{~cm}$, enzyme labeling instrument (Lei she, model: RT-6000), optical microscope (Japanese OLYMPUS, model: BX53), centrifuge (Anhui Jiawen, model: JW3021HR), slicing machine (Thermo Fisher Scientific), Whirlpool mixer (its Limbell instrument manufacturer, model: GL-88B), and in situ apoptosis kit (rusur an n L L experiment. 


\subsection{Model replication}

According to the previous study of our group ${ }^{[9]}$, three special cuboid plexiglass moxibustion boxes (except the bottom edge, each side has four symmetrical holes with a diameter of $10 \mathrm{~mm}$, see Fig. 1), according to the setting of PM2.5 concentration in previous experiments, the moxa smoke environment (high, medium and low moxa smoke concentration) was simulated by opening and closing the holes. Box 1 as a high concentration moxibustion box left 2 holes on the left and right side of the $\mathrm{mg} / \mathrm{m} 3$, box, and the other holes closed to adjust the moxa smoke concentration in the box, while box 2 as a medium concentration moxibustion box left 2 holes on the left, right and top of the $\mathrm{mg} / \mathrm{m} 3$, box, and the other holes were closed to adjust the moxa smoke concentration in the box. Box 3 was used as a low concentration moxibustion box. The concentration of $\mathrm{mg} / \mathrm{m} 3$, box was $0.11 \pm 0.05$.There were 4 holes on the left, right and top of the box, and the rest of the holes were closed. Close the doors and windows of the laboratory, open the indoor ventilation, set the air conditioning temperature at $25^{\circ} \mathrm{C}$, light 3 common moxa bars with a diameter of $1.8 \mathrm{~cm}$, insert them into the moxibustion box, put the moxibustion box in the middle of the three specific moxibustion boxes, and close the door of the box. The moxa smoke concentration in the moxibustion box was detected by the environmental particulate matter sampler. When the moxa smoke PM2.5 concentration reached the corresponding concentration, 10 rats in the low smoke group, middle smoke group and high smoke group were put into the moxibustion box. The rats were observed every 0.5 hours. In order to prevent the "stacking" phenomenon, the rats were forced to move in the cage by beating the moxibustion box with a stick. After the burning of moxa sticks ( $1.5 \mathrm{~h}$ ), ), the new moxa sticks were lit and inserted into the moxibustion box. The time of each fumigation and moxibustion was set at 4 hours. after the experiment, the rats were taken out, put back to the animal room, filled with water, and the window was opened for ventilation. the experiment lasted 3 months, twice a day. The rats in the normal control group were fed without intervention every day during the experiment.

\subsection{Observation indicators and detection methods.}

Because of the death of rats during the process of modeling, eight rats in each group were randomly selected for detection.

The levels of IL-1 $\beta$, IL-6, and TNF- $\alpha$ in the rat serum were detected by ELISA. Briefly, the blood from the abdominal aorta of the rats was placed in an anticoagulant tube at $4^{\circ} \mathrm{C}$ and centrifuged at $3000 \mathrm{rpm}$ for $15 \mathrm{~min}$, and the supernatant was separated. The samples were processed strictly according to the instructions of the corresponding kits for IL-1 $\beta$, IL-6, and TNF- $a$, and the absorbance (OD value) was determined using the enzyme-labeling instrument at a wavelength of $450 \mathrm{~nm}$. The levels of the above indices in serum samples of each group were calculated from the standard curve and then analyzed statistically.

Then, histopathological examination of the nasal mucosa and olfactory bulb was performed. Briefly, after the rats were killed, the nasal mucosa and olfactory bulb of each group were fixed with polyformaldehyde for $48 \mathrm{~h}$, rinsed with distilled water for 10 min, dehydrated in ethanol gradient (50\%, 75\%, $85 \%$, and $95 \%$ ) for $1.5 \mathrm{~h}$, dipped in xylene for $30 \mathrm{~min}$ each time, embedded in paraffin, and sectioned to a thickness of $4 \mu \mathrm{m}$. The sections were then subjected to hematoxylineosin (HE) staining. Briefly, the sections were baked in a constant temperature oven at $65^{\circ} \mathrm{C}$ for 30 min and dewaxed by soaking in xylene I and xylene II for 15 min each. The dewaxed sections were then hydrated by soaking in 100,95, 85, and 75\% ethanol for 5 min each and rinsing for 10 min with tap water each time. The slices were then placed in distilled water and an aqueous solution of hematoxylin for $5 \mathrm{~min}$, followed by color separation in ammonia for 2 min. The slides were then rinsed in running water for $15 \mathrm{~min}$ and dehydrated for 10 min each in $70 \%$ and $90 \%$ ethanol. Then, the slices were stained with ethanol eosin dye 1 for 2 min and dehydrated using absolute ethanol, made transparent by placing them in transparent xylene twice for 3 min each, sealed using neutral gum seal, and put in an oven at $65^{\circ} \mathrm{C}$ for $15 \mathrm{~min}$. Finally, the routine pathological changes of the nasal mucosa and olfactory bulb were observed and photographed under a light microscope.

The apoptotic cells in the nasal mucosa and olfactory bulb of each group were detected by TUNEL staining according to the following procedure: 1) Paraffin sections were dewaxed in water thrice for 10 min each using the standard technique and then washed using PBS. 2) The sections were then digested in trypsin at $37^{\circ} \mathrm{C}$ for $30 \mathrm{~min}$ and washed thrice in PBS for 10 min each. (3) The labeling solution was then added, incubated at $37^{\circ} \mathrm{C}$ for $1 \mathrm{~h}$, and washed using PBS thrice for 10 min each. 4) The sections were then incubated at $37^{\circ} \mathrm{C}$ for 30 min and washed with PBS and AP thrice for 10 min each. 5) The sections were then subjected to NBT/BCIP coloration. (6) Finally, the sections were dehydrated in an alcohol gradient, made transparent in xylene, sealed, and observed under a light microscope. For the negative control, PBS was used instead of labeling solution in step 3.

The olfactory behavior of the rats was analyzed using the buried food pellet test (BFPT) ${ }^{[13]}$.

(1) Training stage: According to the surface $0.5 \mathrm{~cm}$, the rats were put into the restricted rats to find food, and the rats in each group were given adaptive training for three days.

(2) Examination stage: after three days of adaptive training, behavioral tests were conducted on each group. To avoid the influence of circadian rhythm, the experiment began at 6:00-8:00 pm (fasting for $24 \mathrm{~h}$ before the experiment). The feeding time ( $\mathrm{T}$ value), activity distance, and average speed of rats in each group were recorded. A feeding time of more than 300 s indicated impaired olfactory sensitivity of the rats.

\subsection{Statistical analysis}

Statistical analysis was carried out using the statistical software SPSS version 22.0 and GraphPad Prism version 8 . The data were expressed by mean \pm standard deviation $(\varangle x \pm s$ ). If the measurement data were in line with normal distribution and met the homogeneity of variance, the single-factor analysis of variance (one-way ANOVA) was used for the analysis, selecting the least significant difference (least significant difference, LSD) method according to the variance results. For data that were not normally distributed, the non-parametric (rank-sum) test was used. The difference was statistically significant at $p$ -

Loading [MathJax]/jax/output/CommonHTML/jax.js 


\section{Results}

\subsection{Observations of the general state of rats}

Before the experiment, there were no significant differences between the groups in fur appearance, behavior, cage activity, feedback to external stimulation, mental state, second stool, diet, and drinking water. Forty-five days before the rats became active in the smoke environment, the behavioral activity of the rats in the smoking group became yellow, and the amount of water consumed was higher than that in the normal group, and there was no difference in behavioral activity, response to stimulation, mental state, and stool. After 45 days of activity in the smoke environment, there was no significant difference between the low-smoke group and the normal control group, but the activity, the response to stimulus, and the mental state of the rats in the middle-and high-smoke groups were weaker than those in the normal control group.

\subsection{Comparison and analysis of correlation of inflammatory factors in the serum, nasal mucosa, and olfactory bulb of rats in each group detected by ELISA}

Figure 2 respectively shows the levels of IL-1 $\beta$, IL- 6 , and TNF- $\alpha$ in the serum of rats in each group were detected by ELISA. The levels of IL-1 $\beta$, IL-6, and TNF- $\alpha$ in the low, middle, and high-smoke groups were significantly higher than those in the normal control group $(P<0.01)$. There was a positive correlation between the concentrations of proinflammatory factors and smoke $(P<0.01)$. There was a correlation between the concentrations of inflammatory factors in the serum and nasal olfactory bulb $(P<0.0001)$.(Figs. 2)

\subsection{Observations of the structural changes of the olfactory bulb in the nasal mucosa of rats in each group}

The results of the HE staining of the nasal mucosa of rats in each group indicated that the hierarchical structure of the nasal mucosa in the normal control group was clearer and neatly arranged than that in the smoking groups, the surface was covered with cilia, and a few inflammatory cells could be seen under the mucosa. The hierarchical structure of nasal mucosa in the low-smoke group was arranged and neatly covered with cilia, and the number of submucosal inflammatory cells was significantly higher than that in the normal control group. In the middle-smoke group, the mucosal epithelial structure was unclear, disordered, necrotic, and with severe inflammatory cell infiltration. In the high-smoke group, the nasal mucosa was congested and edematous; the structure was unclear and disordered, there were large areas of necrosis, with severe inflammatory cell infiltration under the mucosa. HE staining of the olfactory bulb in each group showed no significant differences between the groups (Fig. 3).

\subsection{Detection of apoptosis in the nasal mucosa and olfactory bulb of rats in each group by TUNEL}

The apoptosis of the nasal mucosa and olfactory bulb of rats in each group was detected by TUNEL staining. Under the microscope, it was observed that the number of apoptotic cells in the low, middle, and high-smoke groups was higher than that in the normal control group, and that in the high-smoke and middlesmoke groups was significantly higher than the low-smoke and normal control groups $(P<0.01)$. There was no significant difference between the low-smoke and normal control groups $(P>0.05)$. (Figs. 4)

\subsection{Observation of the olfactory behavior in rats.}

The results of the buried food experiment revealed a significant difference between the middle-smoke, high-smoke, and the normal control groups in 300 s $(P<$ 0.01), and no significant difference between the low-smoke and normal control groups $(P>0.05)$. The activity distance of rats in each group indicated that the activity of the middle-smoke and high-smoke groups was significantly lower than that of the normal control and low-smoke groups $(P<0.01)$. Comparison between the speed of movement of rats in each group indicated no significant difference between the high-smoke and middle-smoke groups $(P>0.05)$, whereas a significant difference was observed between the high-smoke and normal control or low-smoke groups $(P<0.05)$. (Fig. 5)

\section{Discussion}

The effect of the inhalation of combustion smoke on body respiration has been widely reported, but research on the olfactory system is relatively insufficient. Studies have demonstrated that ${ }^{[14]}$ the damage from inhaling combustion smoke mainly arises from the inhalation of toxic gases in combustion byproducts, such as carbon monoxide, tar, and some ultra-fine particles (PM2.5 or PM10). These substances can enter the body through the olfactory system and cause adverse effects ${ }^{[15-17]}$ such as damaging the innate immune barrier. The effect of inflammatory stimulation on the clearance of respiratory pathogens and toxic particles is caused by the destruction of the normal respiratory epithelium in the body's mucosal immunity and cilia swing, and under the influence of a large number of oxides and free radicals in smoke, the epithelial cells can undergo oxidative stress, induce the apoptosis of olfactory epithelial cells, disrupt the close arrangement of cells, and cause epithelial damage. Furthermore, under long-term smoke stimulation, the inherent ability of the respiratory barriers such as mucosal epithelium and cilia to clear harmful substances is weakened, and the continuous accumulation of pathogens leads to respiratory tract infections and the continuous development of inflammation ${ }^{[18]}$.

It has been reported that ${ }^{[19]}$ smoke can promote the expression of proinflammatory cytokines such as TNF-a, IL-6, IL-1, and IL-8, as well as reduce the content of anti-inflammatory factors such as IL-2 and IL-10. Acute inhalation of combustion smoke even causes pathological changes in the retina, cerebellum, and hippocampus of adult rats ${ }^{[20]}$, as well as increases the vascular permeability and expression of inflammatory factors and chemokines in the olfactory bulb of rats, causing edema and pathological morphological changes to the olfactory bulb ${ }^{[21]}$. The ultra-fine particles constituting PM2.5 may be an important aspect of this process. Studies have demonstrated that ${ }^{[15]}$ ultra-fine particles (PM2.5) can enter the olfactory epithelial cells through the olfactory pathway and then transport to the olfactory bulb, the olfactory cortex, and other brain tissues. The epidemiological investigation also reported that exposure to different

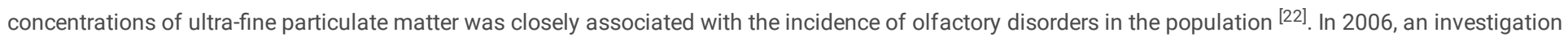

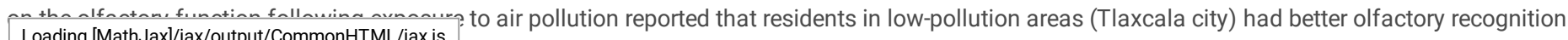


function and that the olfactory function of young people was more easily affected by air pollution compared to that of the elderly population. An earlier report documented that ${ }^{[23-25]}$ the histopathological observation of nasal biopsies in young people in Mexico City with severe air pollution showed basal cell proliferation, squamous metaplasia, and epithelial dysplasia in the front of the middle turbinate. In a subsequent investigation and biopsy, it was observed that apart from the destruction of the olfactory bulb, fine particles were also found in the olfactory nerve and olfactory bulb tissue of residents of the highpollution areas of Mexico City. Similarly, the levels of inflammatory molecules (IL-1 $\beta$ and CD14) in the olfactory bulb also increased significantly. In summary, in the areas that were seriously polluted by air particulates and heavy metals in smoke, the accumulation of fine particles in the olfactory bulb occurs easily and causes a local inflammatory reaction and neuropathological changes, indicating that smoke pollutants can damage the olfactory system through some mechanisms. Studies have demonstrated that ${ }^{[26-27]}$ there are ultrastructural changes in the olfactory mucosa of rats with dysosmia, obvious apoptosis in the olfactory epithelium and lamina propria, thinning of the olfactory epithelium, decrease in the nuclear layer of sensory neurons, and so on.

Moxibustion, mainly through burning moxa hair or columns, directly or indirectly stimulating the acupoints on the body surface, and using the conduction of meridians for the treatment, is an ancient means for the prevention and treatment of diseases in China. Moxibustion has the functions of warming the meridians, invigorating the positive, and dispelling evil. Moxa smoke, which belongs to a type of burning smoke, is produced during the process of moxibustion therapy. A large amount of smoke produced during combustion contains more than 200 chemical components, of which the main products are

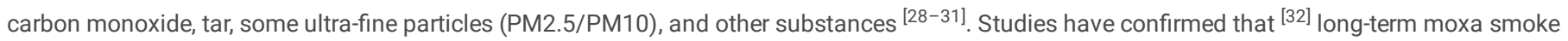
environment often exposes acupuncture practitioners and patients to smoke, and the human body will show varying degrees of eye irritation, cough, and throat discomfort, which can increase the sense of tiredness. It will have a certain impact on people's physique, breathing, olfactory system, suggesting a positive correlation. In a previous study ${ }^{[33]}$, we observed that different concentrations of moxa smoke could change the ultrastructure of the olfactory bulb in rats. This indicates that smoke pollutants can damage the olfactory system through some mechanisms. Hence, this experiment was based on the content of PM2.5 as the standard to distinguish the concentration of environmental particulates in the previous study ${ }^{[10]}$, and low, medium, and high concentrations of moxa smoke were obtained by controlling the content of PM2.5 in a glass moxibustion box. The effects of different concentrations of PM2.5 on the olfactory function and the expression of inflammatory factors in serum, nasal mucosa, and olfactory bulb of rats were analyzed.

As an olfactory-sensitive animal, rats mainly find food through smell. We used a classical olfactory evaluation method (BFPT) to evaluate the rats' sense of smell. By comparing the time taken by the rats in each group to search for food ( $T$ value), it was observed that rats exposed to medium and high concentrations of smoke could not find food within the prescribed 300s and had weak activity and exploration ability. Rats in the normal control group and low-smoke group could find food within the prescribed 300 s and had a strong ability of activity and exploration. From this experiment, we concluded that longterm exposure to medium or high concentrations of smoke will damage the rat's sense of smell and reduce its smelling sensitivity. In the HE sections of the nasal mucosa and olfactory bulb of rats in each group, it was observed that compared to the low-smoke and the normal control groups, the nasal mucosa of the middle and high-smoke groups were congested and edematous, the structure was unclear and disordered, necrotic, and the infiltration of inflammatory cells under the mucosa was more severe, while there was no difference in the HE sections of the olfactory bulb in each group. When the apoptosis of nasal mucosa and olfactory bulb cells was detected by TUNEL, we observed that the apoptosis of nasal mucosa and olfactory bulb cells in low, middle, and highsmoke groups, particularly the middle and high-smoke groups, was significantly higher than that in the normal control group. The results indicated that the moxa smoke environment could damage the inherent structure of the nasal mucosa and olfactory bulb cells of rats, especially at medium and high concentrations of moxa smoke. In a study on the significance of proinflammatory cytokines in the nasal mucosa and olfactory bulb injury, we observed that proinflammatory cytokines such as IL-1 $\beta$, IL- 6 , and TNF- $\alpha$ increased by different degrees in the stress reaction, operation and trauma, and the degree of trauma was correlated with the level of cytokines,Some studies have also demonstrated that ${ }^{\left[{ }^{[3]}\right]}$ TNF-a can destroy the integrity of the porcine respiratory epithelial barrier, which is consistent with the fact that the increased expression of TNF-a in human chronic sinusitis patients can damage the integrity of nasal mucosa epithelium. Moreover, TNF- $\alpha$ can also induce the activation of IL- $1 \beta$, IL-6, and other inflammatory factors. A study ${ }^{[35]}$ compared the nasal mucosa of patients with chronic sinusitis with that of healthy people and reported that IL-1 $\beta$ and IL- 6 were highly expressed in the nasal mucosa of patients with chronic sinusitis. Therefore, we suggest that these three cytokines are associated with nasal mucosal injury. From the results of ELISA, we observed that the expressions of IL$1 \beta$, IL-6, and TNF- $\alpha$ in the serum, nasal mucosa, and olfactory bulb of the low, middle, and high-smoke groups were significantly higher than those of normal control groups, which may be the cause of persistent inflammatory reaction in local tissue, aggravating tissue inflammatory reaction, and affecting the recovery of tissue structure.

The purpose of this study was to evaluate the olfactory behavior of rats, observe the pathological structure and apoptosis of nasal mucosa and olfactory bulb, and monitor the levels and correlations between the proinflammatory factors IL-1 $\beta$, IL-6, and TNF- $\alpha$ in the serum, nasal mucosa, and olfactory bulb of rats in each group, to further explore the effects of different concentrations of PM2.5 smoke on the olfactory function of rats. In the food search experiment, there was no significant difference in the olfactory sensitivity between rats exposed to low concentration of PM2.5 and the normal control group, but in rats exposed to medium and high concentrations of PM2.5, the olfactory sensitivity decreased, the structure of nasal mucosa changed, the apoptosis of nasal mucosa and olfactory bulb cells increased, and the expression of inflammatory factors IL-1 $1 \beta$, IL- 6 , and TNF- $a$ increased. It is suggested that the olfactory function of rats is damaged, and the olfactory sensitivity of rats is decreased by prolonged exposure to a moxa smoke environment with medium and high concentrations of PM2.5.

\section{Conclusions}

In conclusion, the long-term exposure to medium and high concentrations of moxa smoke can decrease the olfactory sensitivity of rats.

\section{Abbreviations}


the buried food pellet test;TNF-a:Tumor necrosis factor-a; IL-6:Interleukin-6; IL-1 $\beta$ :Interleukin-1ß; ELISA:Enzyme-linked immunosorbent assay;PM2.5-10:Coarse Particulate Matter;TUNEL:TdT-mediated dUTP Nick-End Labeling;HE:hematoxylin-eosin staining

\section{Declarations}

\section{Acknowledgements}

Not applicable.

\section{Author contributions}

Prof. Ling Hu and Prof. Zijian Wu provided ideas and designs for this experiment.; Zhiming Jiang completed the whole experiment and received the data; Zhiming Jiang , Xiaojia Li and Lina Zhao analysed the data; Zhiming Jiang led the writing; and Prof. Ling Hu and Prof. Zijian Wu revised the manuscript. All authors have read and approved the manuscript.

\section{Availability of data and materials}

The data used to support the fndings of this study are available from the corresponding author upon request.

\section{Acknowledgments}

This work was supported by the the National Basic Research Program of China,Grant/ Award Number:2015CB554504 and the National Natural Science Foundation of China,Grant/ Award Number: 81503660,The source of funding plays an important role in the design, practice, or analysis of this study.

\section{Ethics approval and consent to participate}

Approval of research protocol:This experiment is an animal Studies has been approved by the Experimental Animal Ethics Committee of Anhui University of Chinese Medicine (Registry and the Registration No. of the study/Trial: 2020028).Informed consent: N/A. The authors declare no Conflict of Interests for this article.

\section{Consent for publication}

Not applicable.

\section{Competing interests}

The authors declare that they have no competing interests

ORCID

Zhiming Jiang ID: https://orcid.org/0000-0003-3117-6263

\section{Author details}

${ }^{1}$ Anhui University of Traditional Chinese Medicine, Acumox And Tuina College; 2 Acumox And Tuina College, Anhui University of Traditional Chinese Medicine, Hefei , China. 3 Research Centre On The Correletion Between Channels And Viscera,Anhui University Of Traditional Chinese Medicine.

\section{References}

1. Song C, Leonard BE. The olfactory bulbectomised rat as a model of depression. Neurosci Biobehav Rev. 2005;29(4-5):627-47. doi:10.1016/j.neubiorev.2005.03.010.

2. Whitman MC, Greer CA. Adult neurogenesis and the olfactory system. Prog Neurobiol. 2009;89(2):162-75. doi:10.1016/j.pneurobio.2009.07.003.

3. Tsuno Y, Kashiwadani H, Mori K. Behavioral state regulation of dendrodendritic synaptic inhibition in the olfactory bulb. J Neurosci. 2008;28(37):922738. doi:10.1523/JNEUROSCI.1576-08.2008.

4. Deutsch CJ, Tan A, Smailes S, Dziewulski P. The diagnosis and management of inhalation injury: An evidence based approach. Burns. 2018;44(5):104051. doi:10.1016/j.burns.2017.11.013.

5. Whitehead GS, Grasman KA, Kimmel EC. Lung function and airway inflammation in rats following exposure to combustion products of carbongraphite/epoxy composite material: comparison to a rodent model of acute lung injury. Toxicology. 2003;183(1-3):175-97. doi:10.1016/s0300483x(02)00542-5.

6. Reczyńska K, Tharkar P, Kim SY, et al. Animal models of smoke inhalation injury and related acute and chronic lung diseases. Adv Drug Deliv Rev. 2018;123:107-34. doi:10.1016/j.addr.2017.10.005.

7. Shirani KZ, McManus AT, Vaughan GM, McManus WF, Pruitt BA Jr, Mason AD Jr. Effects of environment on infection in burn patients. Arch Surg. 1986;121(1):31-6. doi:10.1001/archsurg.1986.01400010037003.

8. Lee HM, Greeley GH, Herndon DN, Sinha M, Luxon BA, Englander EW. A rat model of smoke inhalation injury: influence of combustion smoke on gene Loading [MathJax]/jax/output/CommonHTML/jax.js 
9. Zou YY, Lu J, Poon DJ, et al. Combustion smoke exposure induces up-regulated expression of vascular endothelial growth factor, aquaporin 4, nitric oxide synthases and vascular permeability in the retina of adult rats. Neuroscience. 2009;160(3):698-709. doi:10.1016/j.neuroscience.2009.03.007.

10. Duan Wen-xiu, Ling WuZHu, et al.Air quality safety test in the simulated moxibustion clinic. Zhongguo Zhen Jiu. 2016 Jun 12;36(6):637-640. Chinese. doi: 10.13703/j.0255-2930.2016.06.022.

11. Duan WX, Wu ZJ, Hu L, Song XG, et al. Effect of Different Concentrations of Moxa-smoke on Lung Function and TNF-a and IL-1 $\beta$ Levels in Serum and Lung Tissues in Normal Rats. Zhen Ci Yan Jiu. 2018 Feb 25;43(2):98-103. Chinese. doi: 10.13702/j.1000-0607.170854.

12. Yang J, Zhao BX, Han L, et al. Effects of controllable dynamic inhaled exposure of moxa smoke on LDL-r, ICAM-1 and morphology of heart tissue in rats. Zhongguo Zhen Jiu. 2014 Jun;34(6):573-7. Chinese.doi:.

13. $13703 /$ j.0255-2930.2014.06.014.

14. Edwards DA, Thompson ML, Burge KG. Olfactory bulb removal vs peripherally induced anosmia: differential effects on the aggressive behavior of male mice. Behav Biol. 1972;7(6):823-8. doi:10.1016/s0091-6773(72)80174-3.

15. Guo B, Bai Y, Ma Y, et al. Preclinical and clinical studies of smoke-inhalation-induced acute lung injury: update on both pathogenesis and innovative therapy. Ther Adv Respir Dis. 2019;13:1753466619847901. doi:10.1177/1753466619847901.

16. Calderón-Garcidueñas L, Maronpot RR, Torres-Jardon R, et al. DNA damage in nasal and brain tissues of canines exposed to air pollutants is associated with evidence of chronic brain inflammation and neurodegeneration. Toxicol Pathol. 2003;31(5):524-38. doi:10.1080/01926230390226645.

17. Garcia GJ, Schroeter JD, Kimbell JS. Olfactory deposition of inhaled nanoparticles in humans. Inhal Toxicol. 2015;27(8):394-403. doi:10.3109/08958378.2015.1066904.

18. Pace E, Ferraro M, Di Vincenzo S, et al. Oxidative stress and innate immunity responses in cigarette smoke stimulated nasal epithelial cells. Toxicol In Vitro. 2014;28(2):292-9. doi:10.1016/j.tiv.2013.11.004.

19. Münzel T, Hahad O, Kuntic M, Keaney JF, Deanfield JE, Daiber A. Effects of tobacco cigarettes, e-cigarettes, and waterpipe smoking on endothelial function and clinical outcomes. Eur Heart J. 2020;41(41):4057-70. doi:10.1093/eurheartj/ehaa460.

20. Arnson Y, Shoenfeld Y, Amital H. Effects of tobacco smoke on immunity, inflammation and autoimmunity. J Autoimmun. 2010;34(3):J258-65. doi:10.1016/j.jaut.2009.12.003

21. Zou YY, Kan EM, Cao Q, Lu J, Ling EA. Combustion smoke-induced inflammation in the cerebellum and hippocampus of adult rats. Neuropathol Appl Neurobiol. 2013;39(5):531-52. doi:10.1111/nan.12001

22. Hasegawa-Ishii S, Shimada A, Imamura F. Neuroplastic changes in the olfactory bulb associated with nasal inflammation in mice. J Allergy Clin Immunol. 2019;143(3):978-89.e3. doi:10.1016/j.jaci.2018.09.028.

23. Hudson R, Arriola A, Martínez-Gómez M, Distel H. Effect of air pollution on olfactory function in residents of Mexico City. Chem Senses. 2006;31(1):79-85. doi:10.1093/chemse/bjj019.

24. Calderón-Garcidueñas L, Rodríguez-Alcaraz A, Villarreal-Calderón A, Lyght O, Janszen D, Morgan KT. Nasal epithelium as a sentinel for airborne environmental pollution. Toxicol Sci. 1998;46(2):352-64. doi:10.1006/toxs.1998.2549 .

25. Calderón-Garcidueñas L, Solt AC, Henríquez-Roldán C, et al. Long-term air pollution exposure is associated with neuroinflammation, an altered innate immune response, disruption of the blood-brain barrier, ultrafine particulate deposition, and accumulation of amyloid beta-42 and alpha-synuclein in children and young adults. Toxicol Pathol. 2008;36(2):289-310. doi:10.1177/0192623307313011.

26. Calderón-Garcidueñas L, Franco-Lira M, Henríquez-Roldán C, et al. Urban air pollution: influences on olfactory function and pathology in exposed children and young adults. Exp Toxicol Pathol. 2010;62(1):91-102. doi:10.1016/j.etp.2009.02.117.

27. Wrobel BB, Leopold DA. Olfactory and sensory attributes of the nose. Otolaryngol Clin North Am. 2005;38(6):1163-70. doi:10.1016/j.otc.2005.07.006.

28. Xu X, Shan S, Wang W, Liu H. Analysis of the Components in Moxa Smoke by GC-MS and Preliminary Discussion on Its Toxicity and Side Effects. Evid Based Complement Alternat Med. 2020;2020:2648759. doi:10.1155/2020/2648759. Published 2020 Oct 31.

29. Huang J, Lim MY, Hwang C, Zhao B, Shao L. Contrasts in spatial and temporal variability of oxidative capacity and elemental composition in moxibustion, indoor and outdoor environments in Beijing. Environ Pollut. 2015;202:78-84. doi:10.1016/j.envpol.2015.03.020.

30. Wang X, Yu C, Yang H, et al. Symptoms of long-term exposure to moxa smoke in acupuncturists: a correlation analysis. J Tradit Chin Med. 2018;38(1):132-8. doi:10.1016/j.jtcm.2018.02.009.

31. Huang J, Lim MY, Zhao B, Shao L. PM10 mass concentration and oxidative capacity of moxa smoke. QJM. 2015;108(9):705-10. doi:10.1093/qjmed/hcv008.

32. Yu C, Wang X, Wu QF, Yang J, et al. Epidemiological characteristics of acupuncturists who are sensitive to stimulation of moxa smoke: a face-to-face sectional survey based on 733 participants. Zhongguo Zhen Jiu. 2020 Jan 12;40(1):49-53. Chinese. doi: 10.13703/j.0255-2930.20190705-k0001.

33. Wu Zijian. Effect of moxibustion with Moxibustion on knee osteoarthritis and mechanism of olfaction pathway involved in moxa [D].Anhui University of Chinese Medicine,2017.Chinese.

34. Bercier P, Grenier D. TNF-a disrupts the integrity of the porcine respiratory epithelial barrier. Res Vet Sci. 2019;124:13-7. doi:10.1016/j.rvsc.2019.01.029.

35. Wei L, Zhang Y, Tan H. Changes of TNF-a, PDGF and HA in nasal secretions and olfactory function of patients with chronic sinusitis before and after endoscopic sinus surgery. Exp Ther Med. 2018;16(4):3413-8. doi:10.3892/etm.2018.6611.

\section{Figures}

Loading [MathJax]/jax/output/CommonHTML/jax.js 


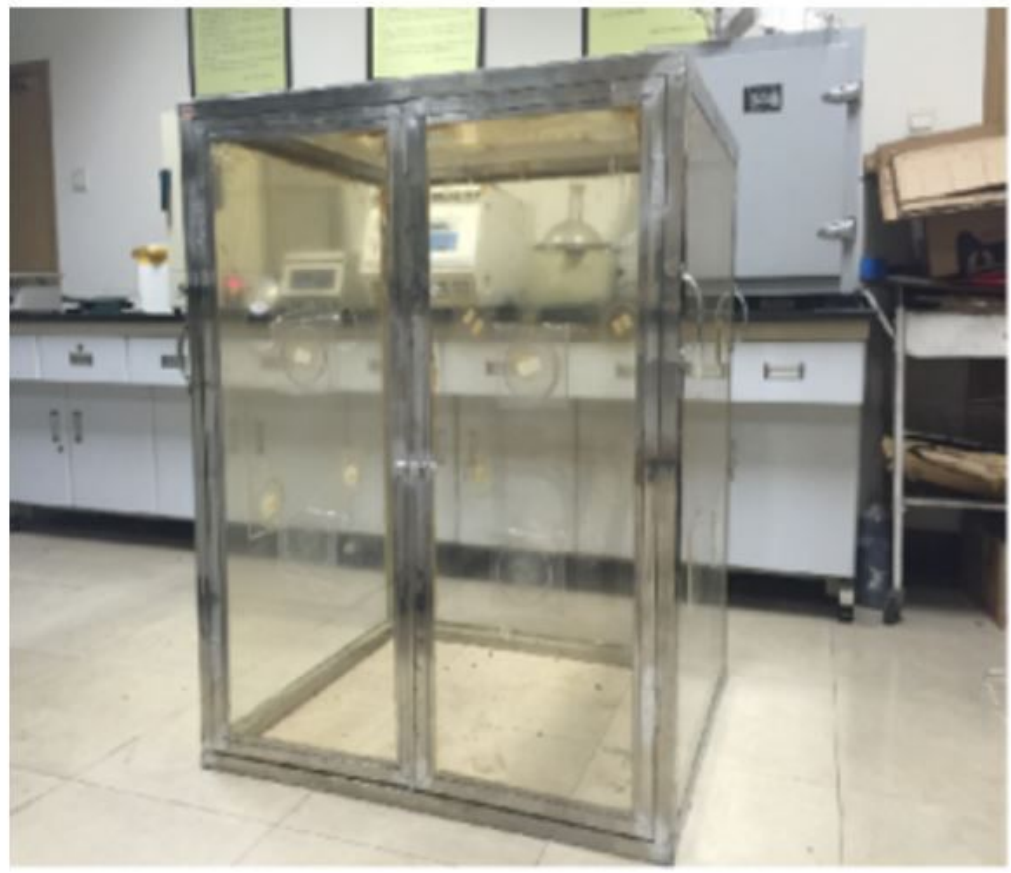

Figure 1

Self-made moxibustion box
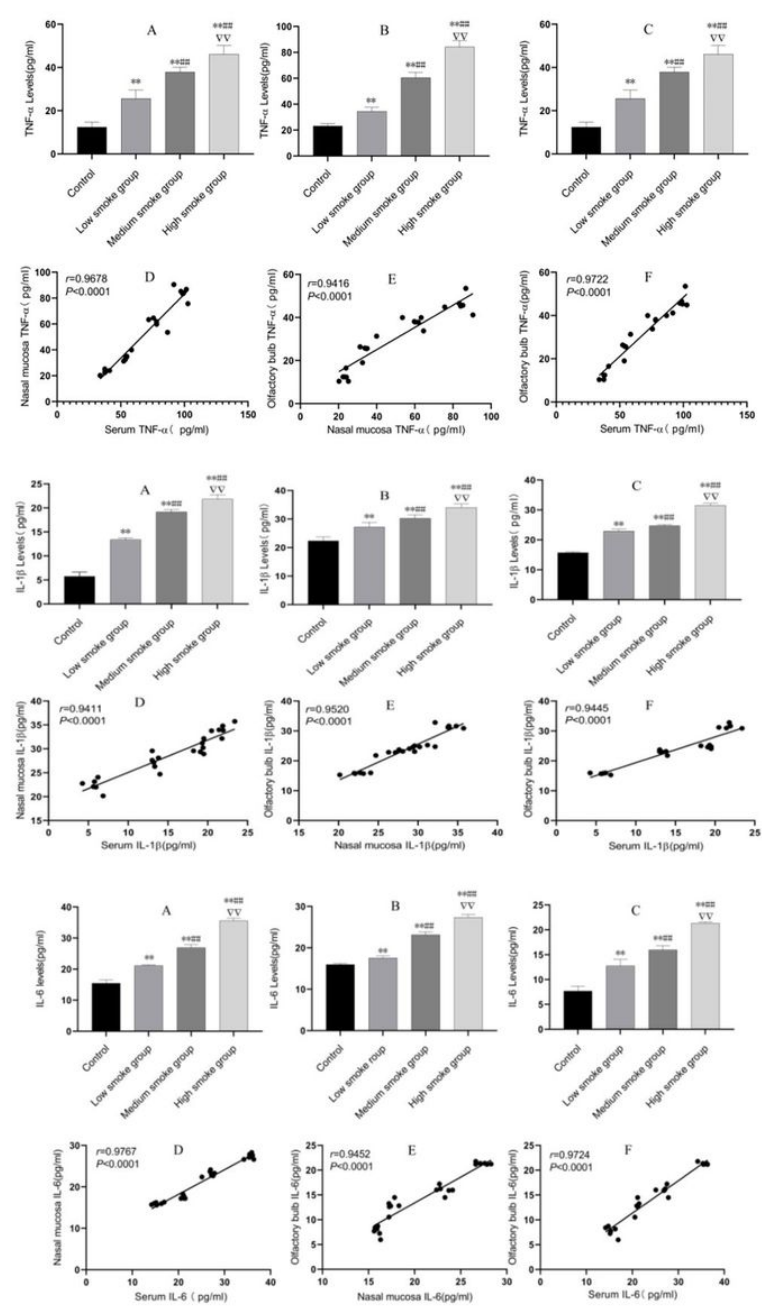


\section{Figure 2}

a Analysis and comparison of the expression of TNF-a and its correlation in the serum, nasal mucosa, and olfactory bulb of rats in each group (

$x \pm s, n=8$ pergroup $).$ Note: $(A, B$, and Crepresentserum, nasal $\mu$ cosa, and olfac $\rightarrow$ rybb, respectively. Thec or relationanalysisofserum, $\mathrm{X} \pm \mathrm{s}, \mathrm{n}=8$ per group). Note: (A, B, and C represent serum, nasal mucosa, and olfactory bulb, respectively. The correlation analysis of serum, nasal mucosa, and olfactory bulb in the Drecoy Epene $\mathrm{F}$ group was compared with the normal group (**P $<0.01$ ), low-smoke group (\#\#P<0.01), and middle-smoke group $(\nabla \nabla \mathrm{P}<$ 0.01). c Analysis and comparison of the expression level of IL- 6 and its correlation in serum, nasal mucosa, and olfactory bulb of rats in each group ( $x \pm s, n=$ 8 per group). Note: (A, B, and C represent serum, nasal mucosa, and olfactory bulb, respectively. The correlation analysis of serum, nasal mucosa, and olfactory bulb in the Drecoy Epene $\mathrm{F}$ group was compared with the normal group ( ${ }^{\star *} \mathrm{P}<0.01$ ), low-smoke group (\#\# $\left.<0.01\right)$, and middle-smoking group $(\nabla \nabla \mathrm{P}<0.01)$.
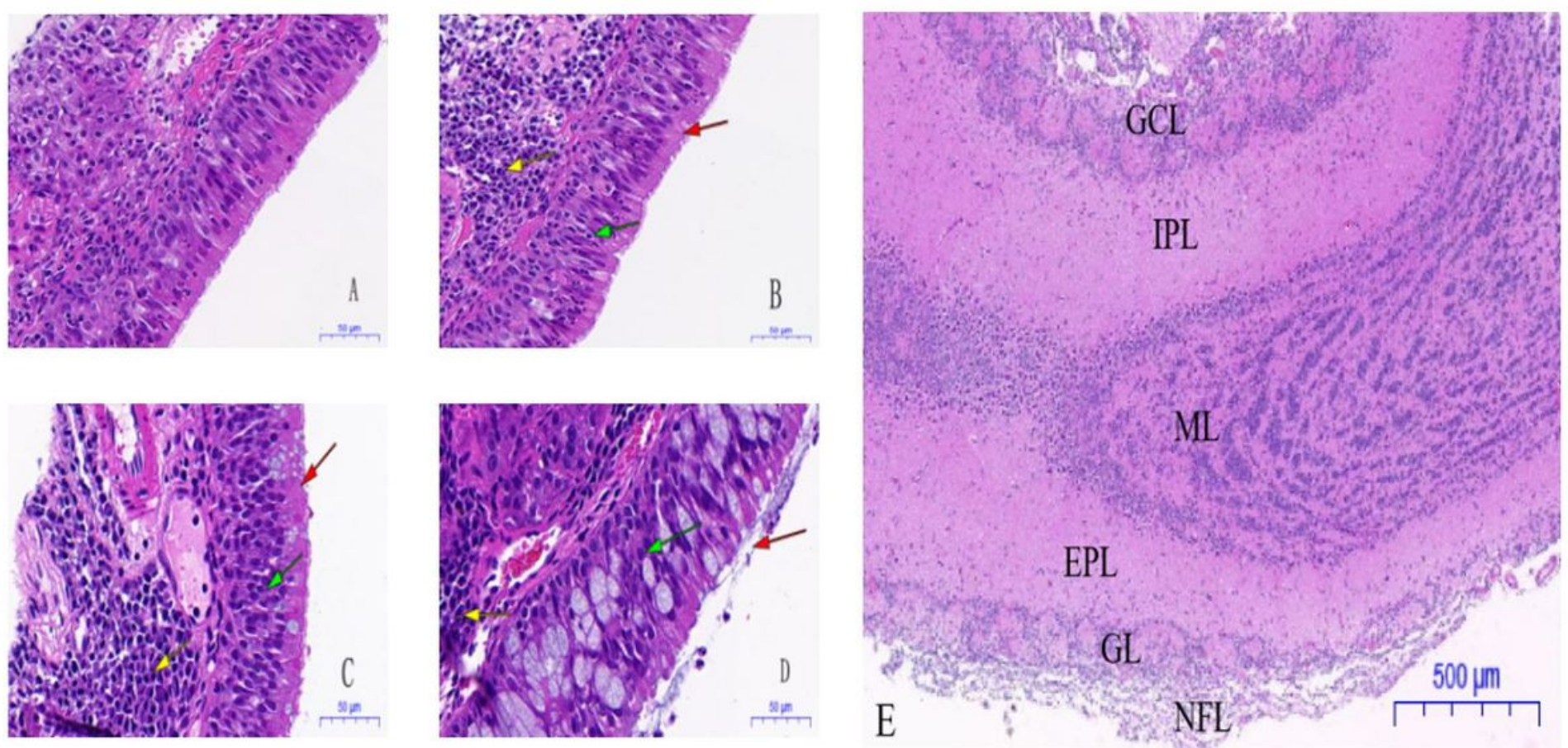

E

\section{Figure 3}

Pathomorphological observations of the nasal mucosa $(\mathrm{HE}, \times 100)$ and morphological observation of the olfactory bulb (HE, $\times 2)$ in each group Note $(1)$ : the pathological sections of the rats in the smoking groups (groups A, B, C, and D represent normal control, low-smoke, middle-smoke, and high-smoke group, respectively) indicated that the columnar ciliated epithelium of nasal mucosa was disordered, with adhesion, even shedding (red arrow), irregularly arranged tissue cells, obvious widening of the intercellular space, thickening of the basement membrane (green arrow), and accumulation of several inflammatory cells (yellow arrow). Scale $=50.0$ um. Note (2): E represents the six well-delineated layers of the olfactory bulb, namely, nerve fiber layer (NFL), glomerular layer (GL), outer plexiform layer (EPL), mitral valve cell layer (ML), inner plexiform layer (IPL), and granular cell layer (GCL). Scale $=500.0 u m$. 

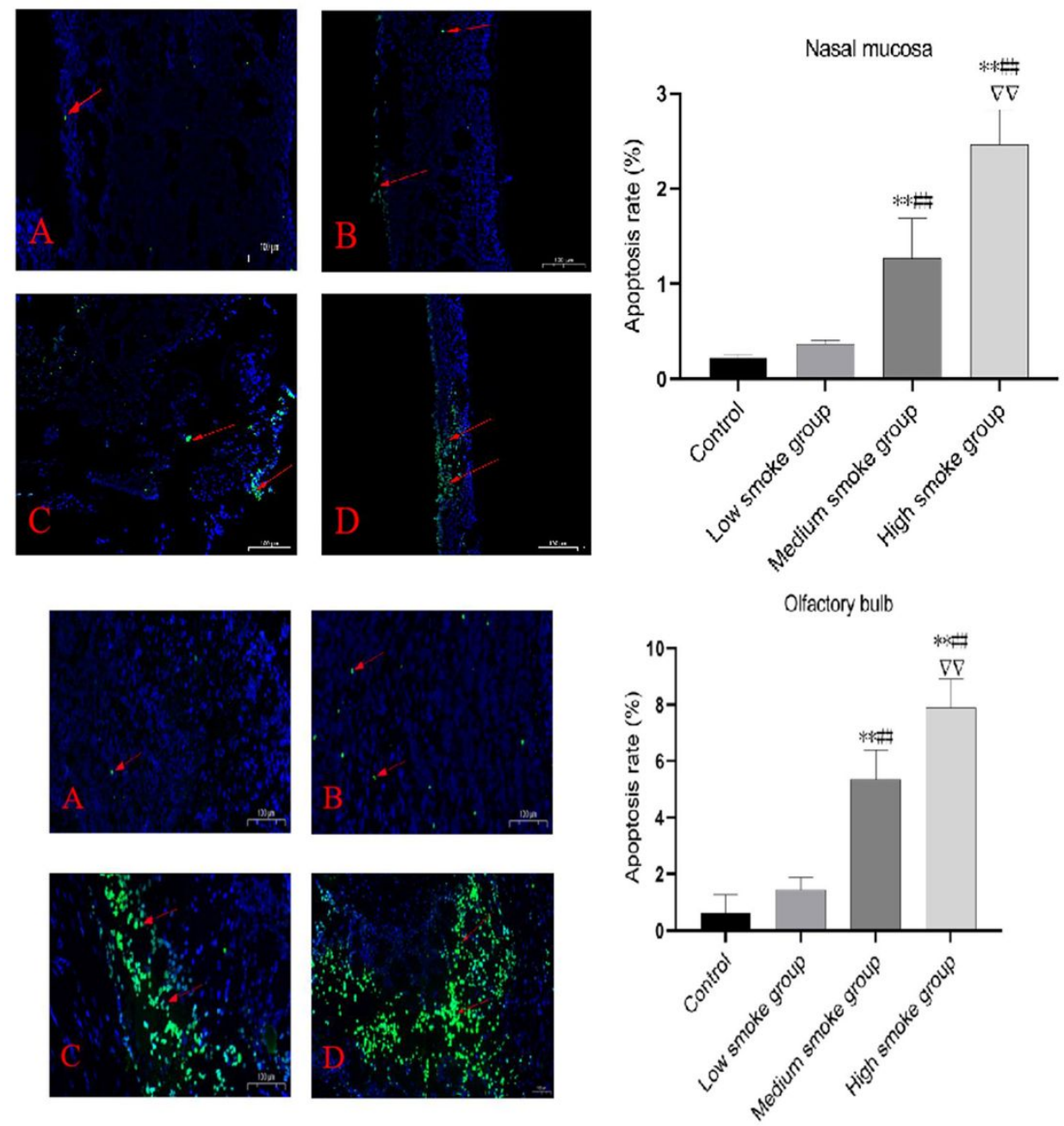

\section{Figure 4}

a Observation of apoptotic cells in the nasal mucosa of rats in each group (TUNEL, $\times 50)$. Note: A, B, C, and D represent normal control, low-smoke, middlesmoke, and high-smoke groups, respectively. Figure 5 left red arrow indicates apoptotic cells; Figure 5 right $E$ shows the statistics of apoptotic cells in the nasal mucosa of rats in each group, compared with the normal control group, $* * P<0.01$, low-smoke group, \#\#P<0.01, and medium-smoke group, $\nabla \nabla \mathrm{P}<$ 0.01. b. Observation of apoptotic cells in the olfactory bulb of rats in each group (TUNEL, $\times 50$ ). Note: A, B, C, and D represent normal control, low-smoke, middle-smoke, and high-smoke groups, respectively. The red arrow indicates apoptotic cells, the right of Figure 6 shows the statistics of the rate of apoptosis of the olfactory bulb in each group, compared with the normal control group, ** $\mathrm{P}<0.01$, low-smoking group, \# \# $\mathrm{P}<0.01$, and middle-smoking group, $\nabla \nabla \mathrm{P}<$ 0.01 . 
A
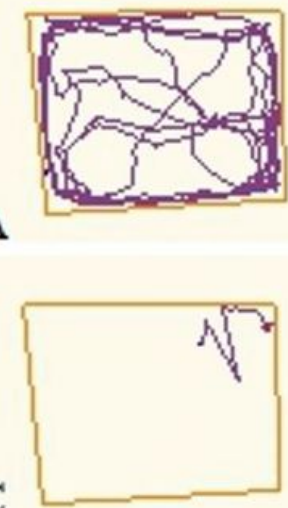

$\mathrm{C}$

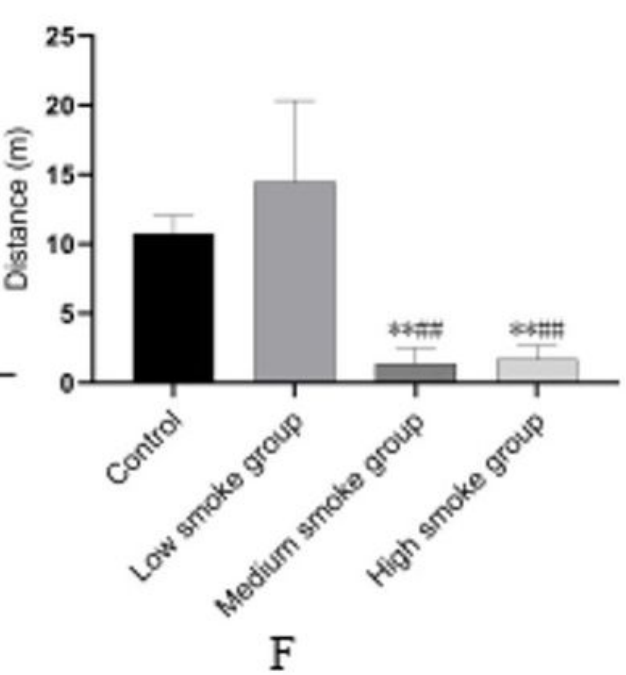

B

D
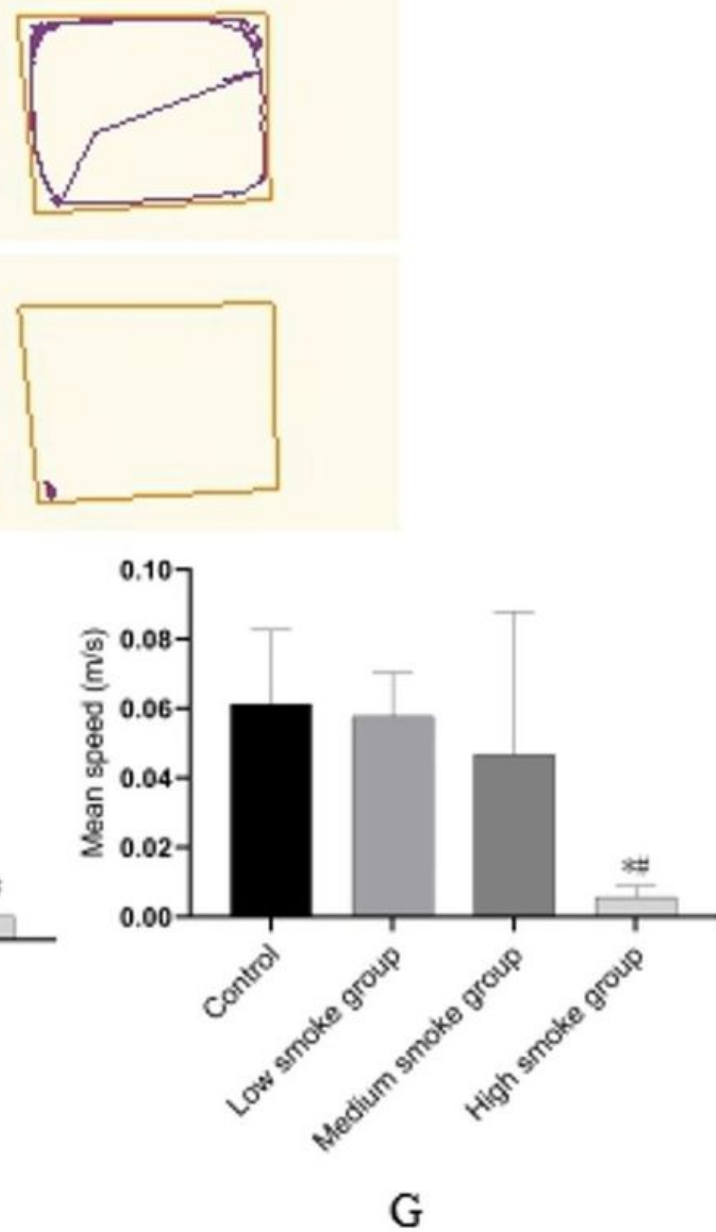

Figure 5

Behavioral observation of rats in each group. Note: A, B, C, and D represent the trajectories of rats in the normal control, low-smoke, middle-smoke, and highsmoke groups, respectively. $(E, F, G)$ shows the statistics of activity time, distance, and average speed of rats in each group, compared with the normal control, ${ }^{*} \mathrm{P}<0.01$, ${ }^{\star \star} \mathrm{P}<0.05$, and the low-smoke group, \# \# $\mathrm{P}<0.01$, \# $\mathrm{P}<0.05$. 